\title{
Preface: Cancer and Stem Cells
}

Accumulated evidence has shown that cancer stem cells (CSCs) have tumor-initiating capacity and play an important role in metastatic relapse and chemoresistance, immunoresistance, and radioresistance. CSC-related markers and functional assays have been used to purify, identify, and therapeutically target CSCs both in vitro and in vivo. Nevertheless, CSC markers are not universal to all tumor types. Additionally, the functional identification based on the specific properties of CSCs is usually limited in in vivo studies. CSCs are situated in a specialized microenvironment (termed "niche"), mainly composed of fibroblasts, endothelial cells, mesenchymal cells, and immune cells, all of which play pivotal roles in resistance. These cells in the microenvironment promote the molecular signaling pathways required for both CSC maintenance and survival and also trigger drug resistance. The stemness pathways that govern CSCs may explain the negative clinical outcomes observed during treatment. Therefore, it is important for oncologists to understand how CSCs contribute to cancer prognosis and how they might be targeted to improve a patient's outcome.

In Chapter 1, titled "Recent Advances in Cancer Stem Cell Targeted Therapy," Akbulut et al. review both the identification and characterization of the CSC population that has been identified in many cancers. They report that the CSC subpopulations may be responsible for the failure of continued treatment in the eradication of cancer due to the high degree of built-in resistance and their intricate role in tumor relapse. The authors suggest, therefore, that therapies targeting the CSC subpopulation are eagerly needed and may be the ultimate aim to eradicate cancer. They propose the potential use of selected unique therapeutics by chemical/biological inhibitors directed at survival/differentiation pathways, including tumor vaccines and checkpoint inhibitors in combination with other therapeutics, to eradicate CSCs. In addition, the authors emphasize the potential use of oncolytic viruses targeting CSCs. This first chapter reviews and summarizes the current state of a variety of therapeutic approaches targeting CSCs.

In Chapter 2, titled "Holistic Approach to Cell Characteristics and Behavioral Analysis," Aktuğ et al. review the field of CSC research and therapies, not only from the therapeutic view of novel strategies and new therapeutic-targeting CSCs, but also from the point of view of a holistic analysis of intracellular and extracellular dynamics and multidisciplinary cooperation among a variety of distinct scientific areas of investigation. The holistic approach suggested by Aktug et al. offers a new mode of therapy and the integration of a variety of disciplines into a unified and centered focus on CSCs.

In Chapter 3, titled "Cancer Stem Cells, Their Microenvironment and Anoikis," Ayla and Karahüseyinoğlu review the roles played by the tissue microenvironment and anoikis in the development and activity of the CSC population. The authors describe how the tumor cells' presence in the tumor microenvironment (TME) face several cell-cell interactions and are signaled by both the interacting cells and by several factors derived from both the tumor cells and the tissues. In this context, the tumor cells face two opposing faces: one that results in tumor cells undergoing a specific death called "anoikis," and another that results in the tumor cells avoiding anoikis and escaping the tumor immune response to result in metastasis at different organ sites. The authors review the underlying mechanisms of the interactions between the tumor cells and the TME interactions; for example, the tumor cell subpopulations that resist anoikis as a consequence of the modulation from the stiffness of the matrix, potentiation of oxidative stress, factors that provide for tumor cell survival, induction of the epithelial to mesenchymal transition (EMT), and self-renewal capability, all of which result in the metabolic deregulation of the cancer cells. The authors also emphasize the role and participation of the perivascular cells, the extracellular matrix, hypoxia, 
etc., all of which regulate CSC plasticity and resistance to anoikis. Such mechanisms should help in the development of new therapies targeting CSCs.

In Chapter 4, titled "The Effects of Lithium Chloride and Midkine on Cancer Treatment," Bilir et al. report on the potential therapeutic application of a combination treatment including inhibitors of midkine (MK), phosphatide inositol-3 kinase (PI3$\mathrm{K}$ ), and glycogen synthase- $3 \beta$ (GSK- $\beta$ ) in combination with lithium chloride in the treatment of tumors, particularly those expressing high levels of PI3-K and GSK3- $\beta$. The rationale of this combination treatment is that many tumors express high levels of PI3-K and GSK3- $\beta$, which promote cell growth and survival, and MK also promotes cell growth, differentiation, survival, and drug resistance. Lithium chloride has been shown to have anti-tumor activity and also is an inhibitor of GSK3- $\beta$. Therefore, it is highly conceivable that this combination treatment may be effective, not only against the bulk tumor population, but particularly against the CSC subpopulation.

In Chapter 5, titled "Cell Signaling Pathways Related to Epithelial Mesenchymal Transition in Cancer Metastasis," Inan and Hayran review the underlying molecular mechanisms by which tumor cells undergo the transition from the epithelial to the mesenchymal phenotype (i.e., the EMT). This phenomenon is observed during embryogenesis and normal stem cell biology. EMT is also observed during cancer progression and the development of metastasis. Under the influence of a variety of complex factors, epithelial tumor cells undergo a series of morphological, cellular, biochemical, molecular, and genetic alterations, leading to their mesenchymal phenotype. The acquisition of the mesenchymal phenotype by certain cancer cells allows them to migrate to distinct organs and then reverse from the mesenchymal to the epithelial phenotype. Several growth factor receptor tyrosine kinases and inflammation-mediated cytokine production all participate in the EMT phenotype. The authors suggest that a thorough understanding of the various mechanisms involved in EMT, particularly in the CSC cell population that is involved in EMT, should help in developing new therapeutics targeting these various mechanisms to avert EMT and reverse resistance.
In Chapter 6, titled "The Importance of Cancer Stem Cells in Gastrointestinal Cancers," Dindar et al. review the high incidence of death rates in patients with gastrointestinal (GI) cancers. Treatment of patients with GI cancers with conventional therapies (surgery, chemotherapy, and radiation) has resulted in clinical responses in a subset of patients, but was not completely satisfactory. Clearly, the new application of immunotherapy, used alone or in combination with conventional therapies, has significantly improved the outcome of patients with GI cancers; however, recurrences and resistance still develop and beg for novel therapies. These unfortunate consequences of the current treatment approaches arose in part as a result of the existence of a subpopulation of CSCs that have the capacity to resist therapies and propagate into metastatic sites. The authors discuss the era of precision medicine and novel technological approaches to investigate the molecular basis of cancer, the role of circulating cancer cells, and the important role of CSCs. It is hoped that a thorough understanding of these cells can lead to the development of new and effective therapies. In particular, the authors discuss the current state of new drugs being developed and tested clinically for GI cancers.

In Chapter 7, titled "Mutualistic Effects of the Myeloid-Derived Suppressor Cells and Cancer Stem Cells in Tumor Microenvironment," Tanriöver and Aytaç discuss the important role of myeloid-derived suppressor cells (MDSCs) in the TME, their close association with CSCs, and how the MDSCs participate in the acquisition of immunosuppression and the metastatic properties of CSCs. The MDSCs derive from myeloid progenitor cells in the bone marrow. Normal myeloid cells differentiate into MDSCs through various mechanisms following the impairment of the normal functional activity of normal myeloid cells. The development of MDSCs occurs in the TME through a complex network of inflammatory mediators. MDSCs then secrete pro-tumorigenic factors such as chemokines, cytokines, and enzymes, which promote tumor cell proliferation, viability, and invasion. Consequently, the MDSCs and CSCs mutually interact with each other and alter tumor cells for unresponsiveness to therapies to promote metastasis.

In Chapter 8, titled "Cancer Stem Cells: Root of the Evil," Erkisa et al. review the pivotal role of the 
minute cell population of CSCs present in the tumor mass as the leading culprit for invasion, metastasis, relapses, and drug resistance. They also discuss similar molecular characteristics of CSCs that resemble mesenchymal cells, resulting in EMT and metastasis. The authors discuss the underlying mechanisms by which CSCs acquire the resistant phenotype and also the roles of ncRNAs in the regulation of the aggressive properties of CSCs. The authors also discuss the potential novel applications to target CSCs.

In Chapter 9, titled "Cancer Stem Cells in Urooncology,"Yüce and Cal review the literature on the resistance and implication of CSCs in urological cancers. The incidence of these cancers increases as a function of the aging population. Conventional treatments for urological cancers include surgery, chemotherapy, radiation, and immunotherapy. Like most other cancers, these treatments do not prevent morbidity or prevent recurrences. In urological cancers, there have been reports that the presence of a subpopulation of CSCs are responsible in large part for treatment failure and death. The authors discuss in detail the properties of CSCs present in bladder, prostate, and kidney cancers with emphasis on means to identifying these CSCs as well as their potential clinical relevance.

In Chapter 10, titled "Cancer and Cancer Stem Cells: New Molecular Perspectives," Gürel et al. provide a novel assessment of the role and clinical implication of CSCs in the majority of cancers and their pivotal roles in invasion, metastasis, and resistance. The authors discuss various perspectives in the field of cancer, such as: (1) alterations of lipid and cholesterol metabolisms and the excess of lipids in CSCs leading to their aggressive and underlying mechanisms of activity; (2) the role of extracellular vesicles (EVs) (exosomes, microvesicles, and apoptotic bodies) that can be transferred among cells and result in promoting tumor angiogenesis, invasiveness, and immune escape (EVs are also involved in CSC niche interactions and can lead to the transformation of normal cells into neoplastic cells); (3) the role of tumor cells in expressing anti-apoptotic genes that prevent the tumor cell's response to death stimuli (such gene products also participate in tumorigenesis); (4) the complex nature of the TME and its pivotal role in the establishment of the CSC niche and acquisition of invasiveness, EMT, and resistant phenotypes; (5) the role of inflammatory cells and their secretion of chemokines/cytokines/growth factors that play important roles in the formation, maintenance, and expression of pluripotent CSCs; and (6) the role of glycosylation encountered in malignant transformation, signaling tumor growth survival and migration, and resistance to both immune and non-immune therapies. The glycosylation patterns seem to maintain the stemness of CSCs and maintain resistance. The authors conclude with the potential therapeutic applications of cancer vaccines and other inhibitors, alone or in combination of other therapies, for the eradication of CSCs.

The CSC field has rapidly grown in the last 25 years, generating thousands of publications per year and this number continues to increase. Furthermore, the use of drugs in lineage tracing, single-cell analysis, and organoid cultures, alone or in combination, will represent important tools to gain new insights regarding the complexity of the CSC biology and will allow pharmacologic companies to test new drugs to target cancer cell stemness.

This special issue of Critical Reviews ${ }^{\mathrm{TM}}$ in Oncogenesis consists of 10 chapters that review the field of CSCs from several points of view, including biology, biochemistry, genetics, and targeting effects. Clearly, this discussion of CSC research is timely and this issue contributes knowledge on the state of art of CSCs and new approaches to targeting them for better clinical responses. In particular, this issue will be used as a reference for both scientists and clinicians.

The Editor-in-Chief wishes to thank the Guest Editor, Dr. Gulperi Oktem, for her diligent effort in selecting the various contributors in this issue. The Editor-in-Chief specifically acknowledges the significant contribution made by Ms. Jazelle Bautista in the development, editing, and formatting of this special issue.

Benjamin Bonavida, $\mathrm{PhD}$

Editor-in-Chief

Department of Microbiology, Immunology and Molecular Genetics

David Geffen School of Medicine

University of California at Los Angeles

Los Angeles, California, USA 
